\title{
Diseño, construcción e implementación de un sistema automatizado integrador para los módulos de caudal, presión y temperatura del centro de manufactura avanzada
}

\begin{abstract}
RESUMEN
El artículo presenta una alternativa para la industria manufacturera y de procesos, siendo el objetivo principal el de integrar los módulos automatizados de caudal, presión y temperatura del Centro de Manufactura Avanzada (CEMA-UNMSM), mediante microcontroladores, respondiendo a la necesidad del sector empresarial, de mejorar su productividad haciendo uso de tecnología económica a fin de incrementar la calidad de sus productos y reducir costos de fabricación.

El resultado logrado en la investigación es la integración de los sistemas de control de procesos del CEMA, que trae como conclusión la factibilidad de realizar dispositivos funcionales de reducido espacio de instalación, versátil en el proceso y de fácil operación mediante software.
\end{abstract}

Palabras clave: Microcontrolador, Instrumentación, Interfaz

DESIGN, CONSTRUCTION AND

IMPLEMENTATION OF AN AUTOMATED

SYSTEM INTEGRATION FLOW, PRESSURE AND TEMPERATURE MODULES FOR

CENTRO DE MANUFACTURA AVANZADA

\section{ABSTRACT}

The article presents an alternative for manufacturing and process industry, aims to integrate flow, pressure and temperature modules automated for Centro de Manufactura Avanzada (CEMA-UNMSM) through Microcontroller, responding to the need of the business sector, to improve their productivity by using economic technology to increase product quality and reduce manufacturing costs.

The results achieved in the research is the integration of process control systems for CEMA, which brings in conclusion the feasibility of functional devices reduced installation space, versatile and easy process operation using software.

Keywords: Microcontroller, Instrumentation, Interface

\section{INTRODUCCIÓN}

El incremento competitivo en el mercado de la industria electrónica, crea la necesidad de diseñar sistemas con mejores características, de menor tamaño, bajos requerimientos de energía, mejor realización, teniendo en especial énfasis sobre todo en la facilidad de la duplicidad del sistema diseñado. La lógica definida por el usuario (cliente) y realizada por el fabricante, permite individualizar a los sistemas diseñados, así como también apegarse más a los requerimientos específicos del usuario (cliente). Esto tiene repercusión en los costos, realización, compactibilidad, desempeño y seguridad del diseño,

Un microcontrolador es un sistema electrónico dentro de un pequeño dispositivo, lo cual ofrece un enorme panorama hacia el mundo de la competitividad. En estos circuitos el usuario puede programar, en un solo chip, para implementar y desarrollar múltiples aplicaciones y funciones con menos componentes de la circuitería tradicional de compuertas, optimizando el espacio físico de los mismos, más económicos, y sin la limitación de que un cambio de lógica del usuario, ocasione un cambio en el diseño del hardware, ya que, los cambios o ampliaciones futuras son casi nulos.

El avance de la tecnología ha motivado a que las empresas apliquen las diferentes metodologías para automatizar y controlar sus procesos, aumentando su productividad y competitividad, constituyéndose en un factor clave para el éxito empresarial (1).

En los últimos años, la tecnología para la automatización industrial viene evolucionando vertiginosamente, de tal manera que el monitoreo y control en tiempo real de las variables de los procesos, para lo cual se utilizan distintos periféricos, software de aplicación, sistemas remotos, etc., que permiten al operador visualizar y tener el completo acceso al proceso haciendo uso del computador, laptop, palm, tablet o celular, resultando soluciones costosas propias de la gran empresa; sin embargo, el presente proyecto permite, realizar un prototipo $70 \%$ más económico e integrado al proceso mediante microcontroladores.

\footnotetext{
Ingeniero Industrial UNMSM. Especialista de la Oficina de Investigación Científica y Desarrollo Tecnológico de la Marina de Guerra del Perú.

E-mail: jorgeepd@hotmail.com

2 Ingeniero Industrial UNMSM. Jefe del Centro de Manufactura Avanzada - CEMA UNMSM E-mail: eraffolecca@yahoo.es

3 Bachiller en Ingeniería Industrial UNMSM. Investigadora del Centro de Manufactura Avanzada - CEMA UNMSM.
}

E-mail: liliana.berrospi@gmail.com 


\section{DESCRIPCIÓN DE LA PLANTA DE CONTROL DE PROCESOS DEL CENTRO DE MANUFACTU- RA AVANZADA}

El Centro de Manufactura Avanzada CEMA de la Facultad de Ingeniería Industrial de la Universidad Nacional Mayor de San Marcos; es una moderna unidad dedicada a la Investigación, capacitación y desarrollo de proyectos relacionados a la manufactura, automatización industrial e inteligencia artificial. (2)

La planta de control de procesos opera mediante los módulos de caudal, presión y temperatura controlando sus variables de manera independiente.

\section{A. PLANTA DE CONTROL DE CAUDAL}

En esta planta se controla el flujo de líquidos no agresivos impulsado por una bomba centrífuga y recorre todo el circuito cerrado de tuberías, cuyo control se realiza mediante una válvula de control proporcional que regula el fluido que pasa a través de ella. (3)

\section{Características Técnicas}

- Dimensiones : $132 \times 50 \times 165 \mathrm{~cm}$

- Estructura sobre ruedas en hierro cromado.

- Sistema de tuberías de $3 / 4$ "en acero inoxidable AISI 316.

- Líneas de conexión y válvulas de cierre en acero inoxidable AISI 316.

- 1 tanque de recogida de agua en acero inoxidable AISI 316, capacidad: 30,8 litros.

- 1 bomba centrífuga $Q=20-80 \mathrm{l} / \mathrm{min}$, con todas sus partes húmedas en acero inoxidable AISI 316 , altura de elevación 30.7 - 23.5 m.c.a, motor de $0.92 \mathrm{Kw}$.

- 2 interruptores de nivel en polipropileno, presión máxima : 10 bar

- 1 transmisor de caudal, rango: 0 a 140 LPM, con una salida de 4 - $20 \mathrm{~mA}$.

- 1 convertidor de frecuencia, voltaje: 200 - 240 VAC, potencia: 0,75 Kw (1 HP).

- 1 tablero electrónico que incluye pulsadores de arranque y parada, botones indicadores de funcionamiento, seleccionador de operación manual - automático.

- 1 PLC Allen Bradlley con módulos de entrada y salida analógica.

- 1 registrador digital.
- Botón de Parada de Emergencia.

- 1 llave de alimentación principal, 1 contactor, 1 fuente de alimentación 24 DVC, 1 bornera de conexiones y 1 portafusibles.

- Rango de operación : (0 a 85) LPM (Óptimo)

Figura 1. Esquema del módulo de caudal

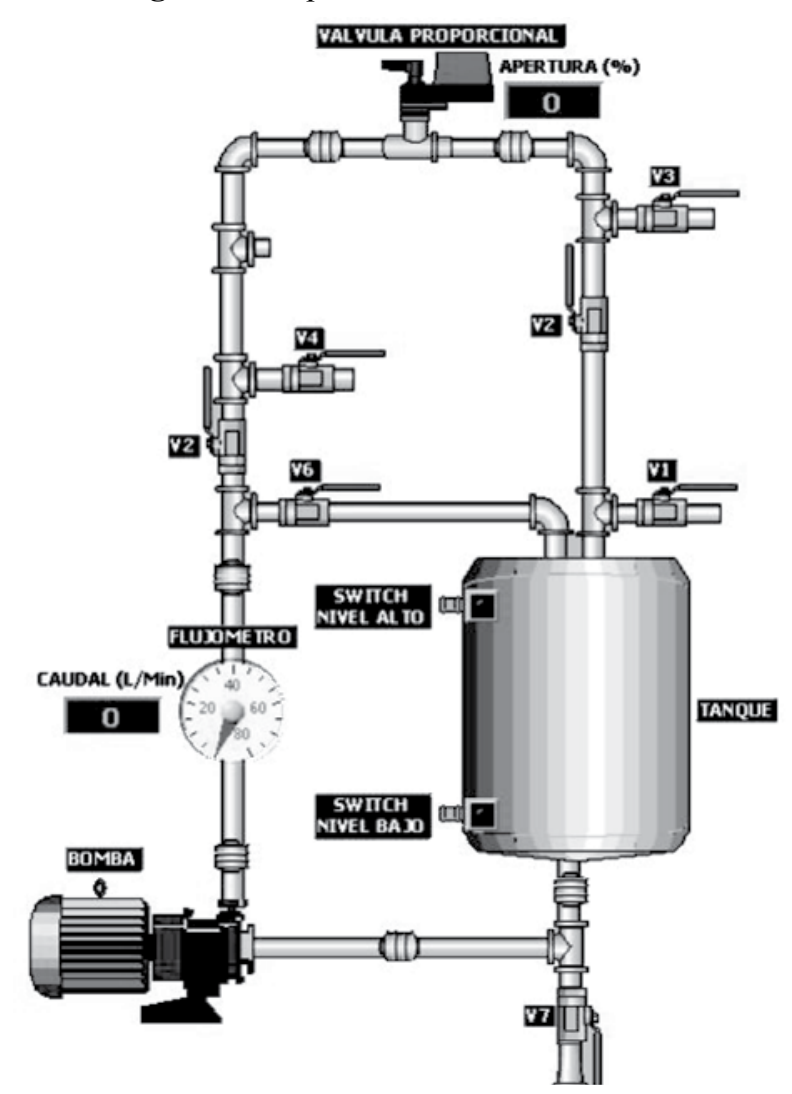

Fuente: Manual del Módulo de Caudal INDUCONTROL

\section{B. PLANTA DE CONTROL DE PRESIÓN}

En esta planta se controla la presión del aire en el interior de un tanque. Una válvula regula la presión del aire que entra al tanque, el cual pasa por un rotámetro, y luego a una válvula neumática que regula la presión del aire de alimentación que ingresa al tanque, mientras el sensor de presión indica la presión del aire en el interior del tanque. (4)

\section{Características técnicas}

- Dimensiones : $132 \times 50 \times 165 \mathrm{~cm}$ Dimensiones : $174 \times 50 \times 165 \mathrm{~cm}$

- Estructura sobre ruedas en hierro cromado.

- Sistema de tuberías de $3 / 4$ " en acero inoxidable AISI 316.

- Líneas de conexión y válvulas de cierre en acero inoxidable AISI 316.

- 1 tanque de recogida de agua en acero inoxida- 
ble AISI 316, capacidad: 30,8 litros.

- 1 tanque presurizado en acero inoxidable AISI 316 , capacidad 20,4 litros.

- 1 bomba centrífuga $\mathrm{Q}=2,5 \mathrm{~m} 3 / \mathrm{hr}$, con todas sus partes húmedas en acero inoxidable AISI 316 , altura de elevación 34 m.c.a, motor de $0,75 \mathrm{HP}$.

- 1 Transmisor de presión rango: 0 - 6 bar, señal de salida 4 - $20 \mathrm{~mA}$.

- 1 válvula de alivio de presión a 5 bar.

- 1 válvula de control caracterizado tipo bola de 2 vías, $C V=4,7 \mathrm{DN}=20$.

- 1 manómetro en acero inoxidable AISI 316, rango : 0 - 6 bar.

- 2 interruptores de nivel en polipropileno, presión máxima : 145 psi.

- 1 presostáto (control de presión), rango -0,2 - 8 bar.

- 1 convertidor de frecuencia, voltaje: $200-240$ VAC, potencia: 0,75 Kw (1 Hp).

- 1 tablero electrónico que incluye pulsadores de arranque y parada, botones indicadores de funcionamiento, seleccionador de operación manual - automático.

- 1 PLC con módulos de entrada y salida analógica.

- 1 registrador digital de tipó HMI.

- 1 llave de alimentación principal, 1 contactor, 1 fuente de alimentación 24 DVC, 1 bornera de conexiones y 1 portafusibles.

- Rango de operación: (5-40) Psi (Óptimo)

\section{PLANTA DE CONTROL DE TEMPERATURA}

Con este módulo automatizado se puede controlar la temperatura del agua mediante dos tanques concéntricos de agua a diferentes temperaturas.

En el primer tanque se calienta el agua a través de una resistencia, hasta llegar a la temperatura deseada.

En el segundo tanque se mezcla el agua fría con el agua caliente del primer tanque.

La válvula proporcional regula el flujo de agua caliente de un tanque al otro para lograr la temperatura deseada con la mezcla según indicaciones del PLC, que actúa según los valores captados por el sensor de temperatura de cada tanque. (5)
Figura 2. Esquema del Módulo de Presión

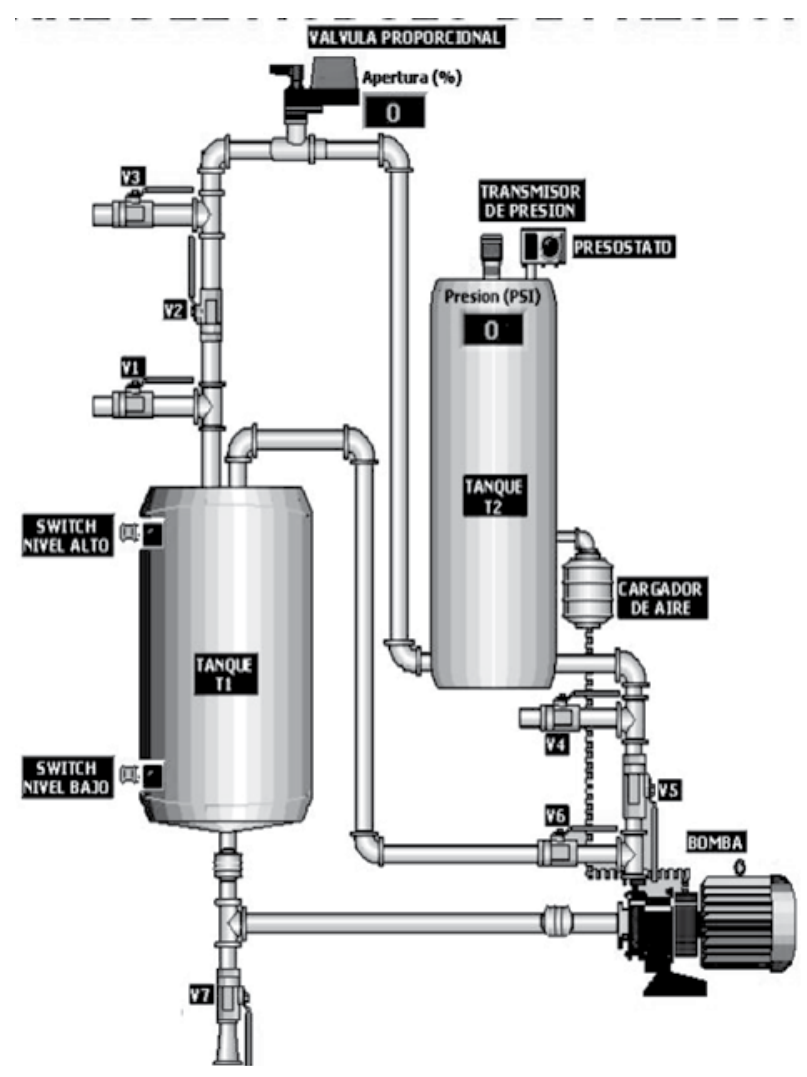

Fuente: Manual del Módulo de Presión INDUCONTROL

\section{Características técnicas}

- Dimensiones : $132 \times 50 \times 165 \mathrm{~cm}$ Dimensiones : $174 \times 50 \times 165 \mathrm{~cm}$

- Estructura sobre ruedas en hierro cromado.

- Sistema de tuberías de 3/4 "en acero inoxidable AISI 316.

- Líneas de conexión y válvulas de cierre en acero inoxidable AISI 316.

- 1 tanque de recogida de agua en acero inoxidable AISI 316, capacidad : 30,8 litros.

- 1 tanque presurizado en acero inoxidable AISI 316 , capacidad 20,4 litros.

- 1 bomba centrífuga $\mathrm{Q}=2,5 \mathrm{~m} 3 / \mathrm{hr}$, con todas sus partes húmedas en acero inoxidable AISI 316, altura de elevación 34 m.c.a, motor de $0,75 \mathrm{HP}$

- 1 Transmisor de temperatura rango: 0 - 6 bar, señal de salida 4 - $20 \mathrm{~mA}$.

- 1 válvula de control caracterizado tipo bola de 2 vías, $C V=4,7 \mathrm{DN}=20$.

- 1 manómetro en acero inoxidable AISI 316, rango : 0 - 6 bar. 
- 2 interruptores de nivel en polipropileno, presión máxima: 145 psi.

- 1 convertidor de frecuencia, voltaje: $200-240$ VAC, potencia: 0,75 Kw (1 HP).

- 1 tablero electrónico que incluye pulsadores de arranque y parada, botones indicadores de funcionamiento, seleccionador de operación manual - automático.

- 1 PLC con módulos de entrada y salida analógica.

- 1 registrador digital con touchscreen.

- 1 llave de alimentación principal, 1 contactor, 1 fuente de alimentación 24 DVC, 1 bornera de conexiones y 1 portafusibles.

- Rango de operación: (3-45)º C (Óptimo)

Figura 3. Esquema del módulo de temperatura

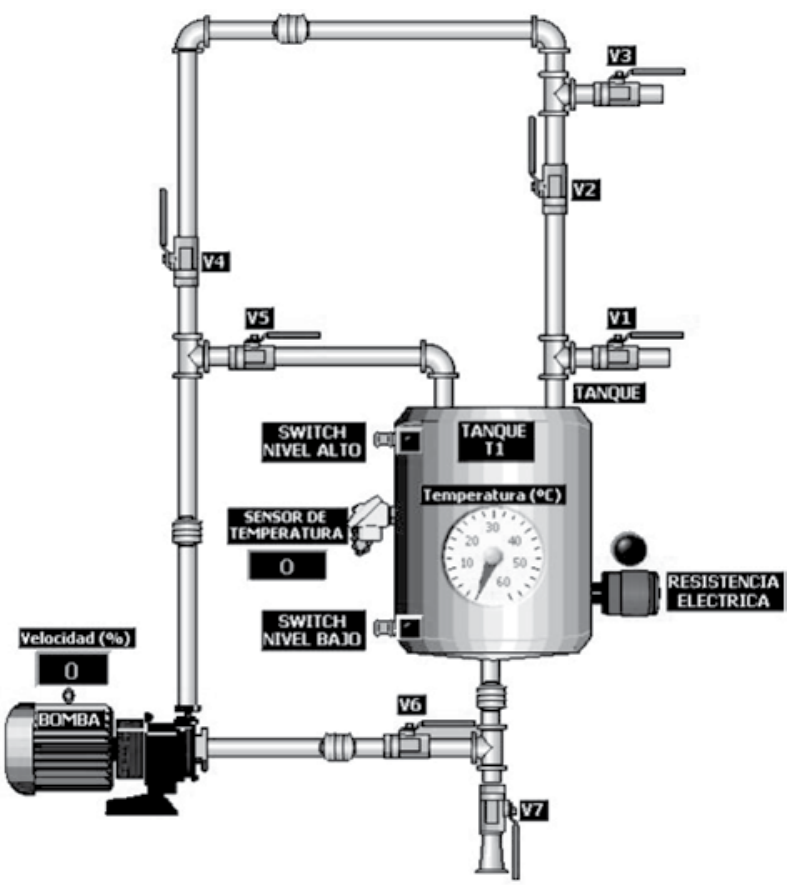

Fuente: Manual del Módulo de Temperatura INDUCONTROL

\section{SOLUCIÓN DE INTEGRACIÓN DESARRO- LLADA EN LOS MÓDULOS}

Se diseño un sistema cuyas características son los siguientes:

- El sistema brinda conexión entre los sistemas de control de cada proceso y reporta el estado del proceso a una central de monitoreo que visualiza los parámetros mediante la aplicación SCADA.

- Cada tarjeta de interfaz se encarga de colectar los diferentes parámetros de cada proceso y los almacena para luego reportarlos al Concentrador eSAT_USB2.0, esta transmisión es mediante el bus eSAT485.

- El concentrador a su vez se encarga de administrar la comunicación con cada tarjeta de interfaz, ordena, almacena, agrupa transmite al ordenador central mediante comunicación USB2.0.

- La transferencia de datos se da a través del software SCADA instalado en el ordenador central.

- Desde el interfaz gráfico del Software SCADA se puede monitorear cada uno de los procesos que forman parte de la red.

Componentes principales

- (3) Tarjeta de Interfaz

- 1) Bus 485 optimizado

- (1) Concentrador

- (1) Aplicación SCADA

Figura 4. Diagrama del módulo integrador

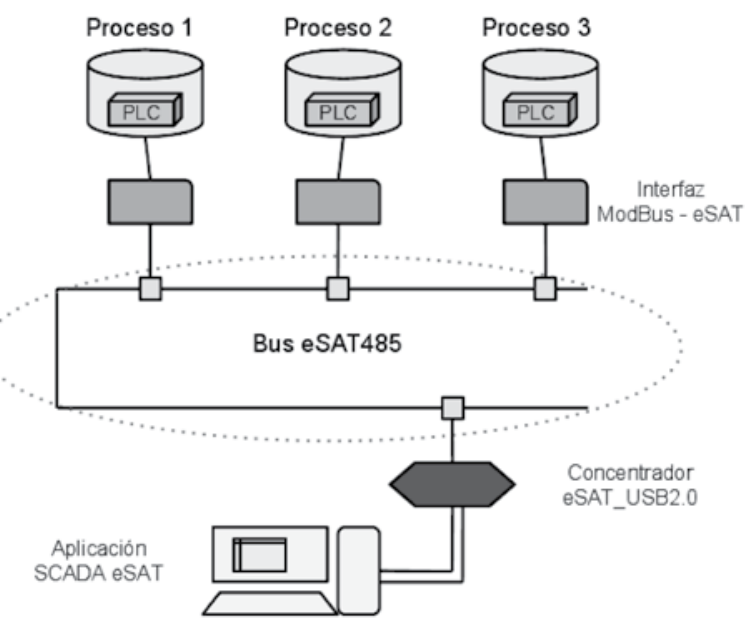

Fuente: Elaboración propia

Adicionalmente, la instalación del sistema implica la instalación de canaletas y demás módulos de soporte para las tarjetas y el bus eSAT485 de la empresa Equipment \& Systems for Advanced Technology S.A.C., representado en los siguientes esquemas simulados con el software Eagle 6.2.

\section{Etapas del circuito}

\section{a. Etapa de Control}

En esta etapa se trabaja con el Microntrolador PIC $18 F 4550$ de la empresa Microchip Inc. y controla la información recibida por los módulos de caudal, presión y temperatura. 
Figura 6. Etapa de control del módulo integrador

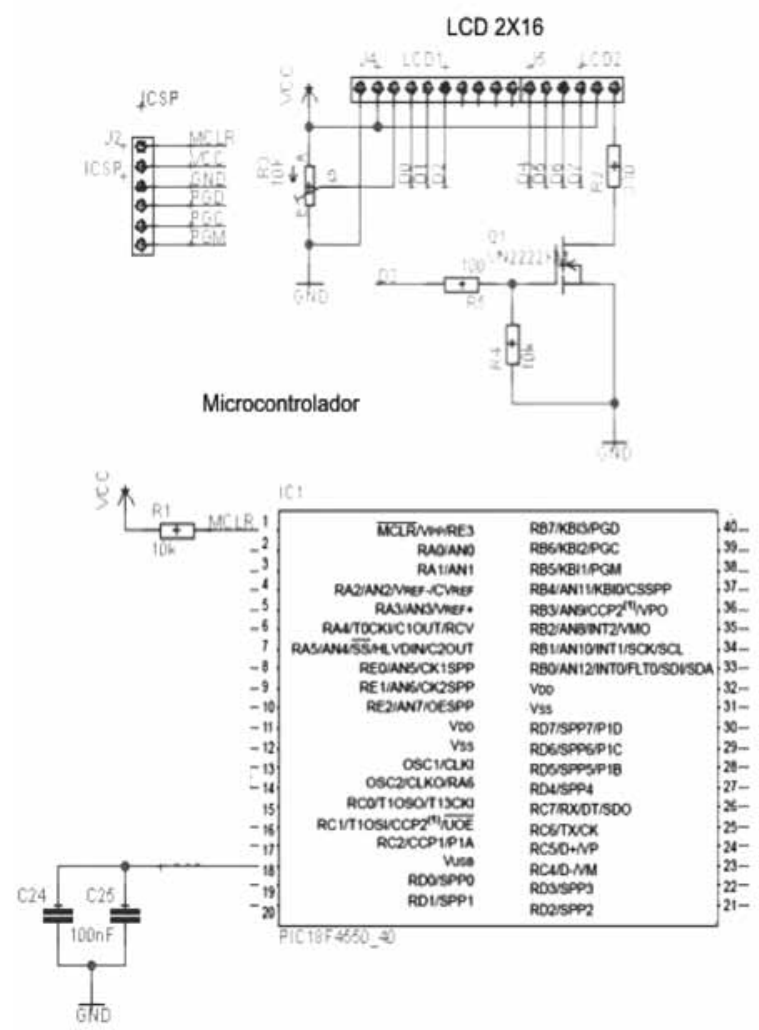

Fuente: Elaboración propia

\section{b. Etapa de control de potencia}

En esta etapa se controla el voltaje deseado para los diversos componentes del circuito

Figura 7. Etapa de control del módulo integrador

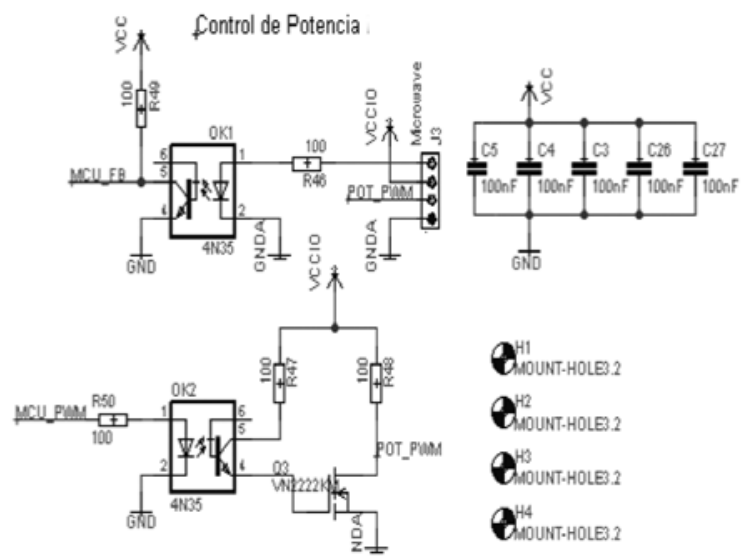

Fuente: Elaboración Propia

\section{c. Etapa de comunicación serial}

En esta etapa se realiza la adquisición de datos de los PLC provenientes de los módulos transformando su señal MODBUS en RS 485 y posteriormente a USB
Figura 8. Etapa de comunicación serial del módulo integrador
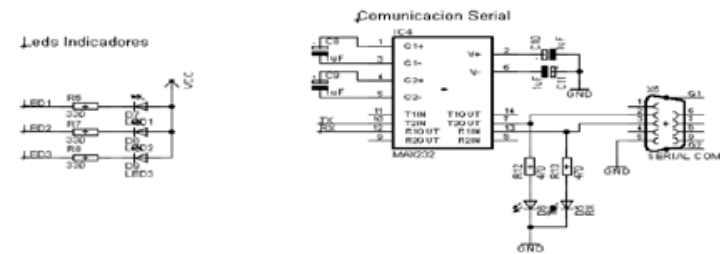

Fuente: Elaboración Propia

\section{d. Etapa de alimentación}

En esta etapa se dota de energía a todo el circuito, y se realiza por medio de una fuente de alimentación y botones funcionales selectores que pueden ser activados de manera manual o automática.

Figura 9. Etapa de alimentación del módulo integrador
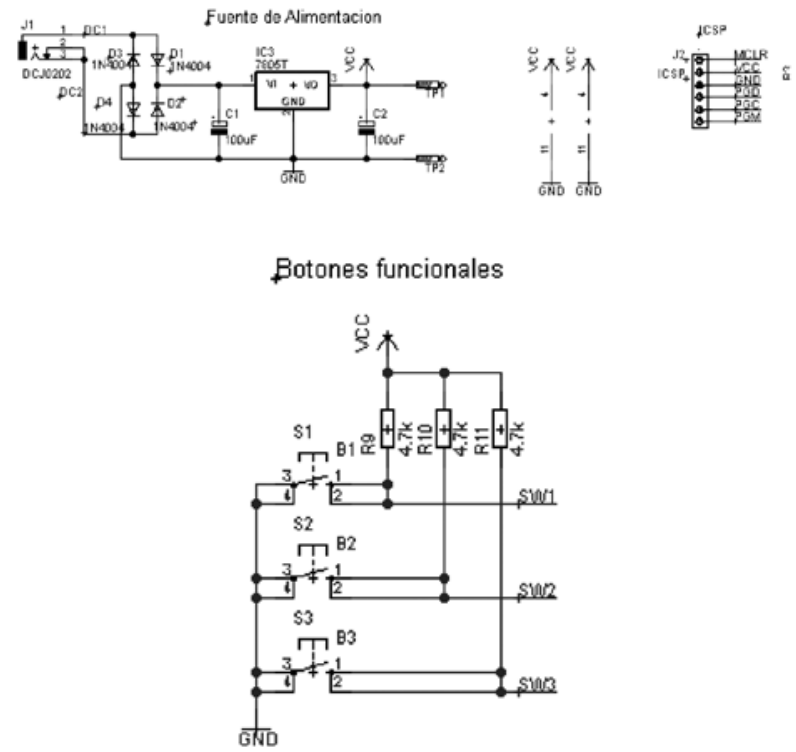

Fuente: Elaboración Propia

\section{Diseño de la tarjeta integradora}

Con las etapas anteriores, se procede a realizar la simulación de los circuitos en la tarjeta final, utilizando para tal fin el software Eagle 6.2

Figura 10. Diseño de la tarjeta integradora

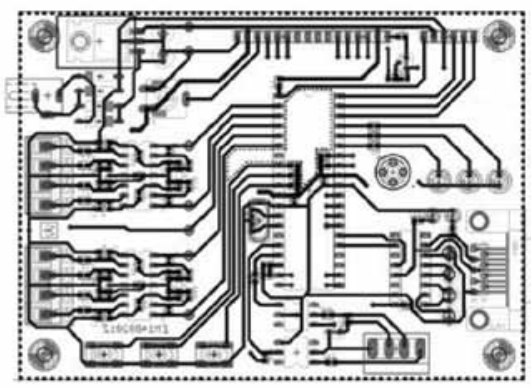

Fuente: Elaboración Propia 


\section{CONSTRUCCIÓN DE LA TARJETA INTEGRA- DORA}

Se insertaron los componentes que se aprecian en el circuito, teniendo como resultado final, el siguiente:

Figura 11. Vista frontal de la tarjeta integradora

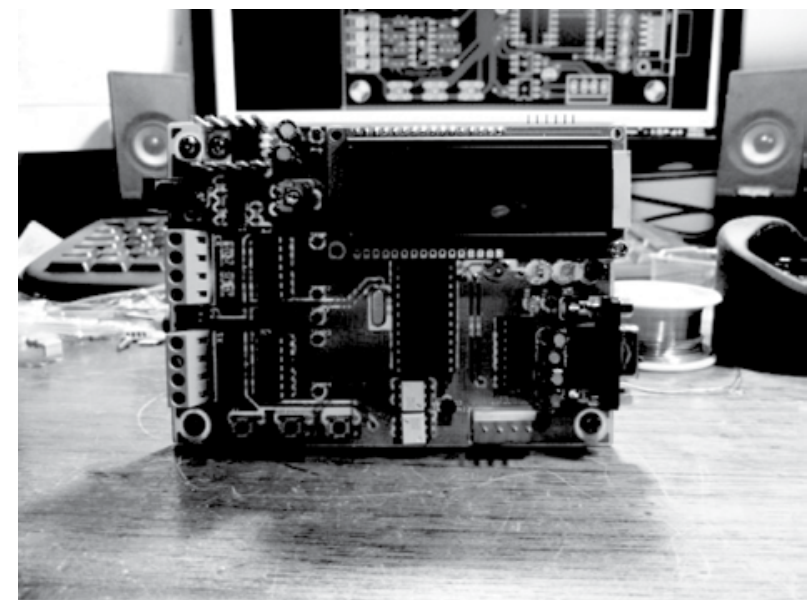

Fuente: Elaboración Propia

Figura 12. Pistas eléctricas de la tarjeta integradora

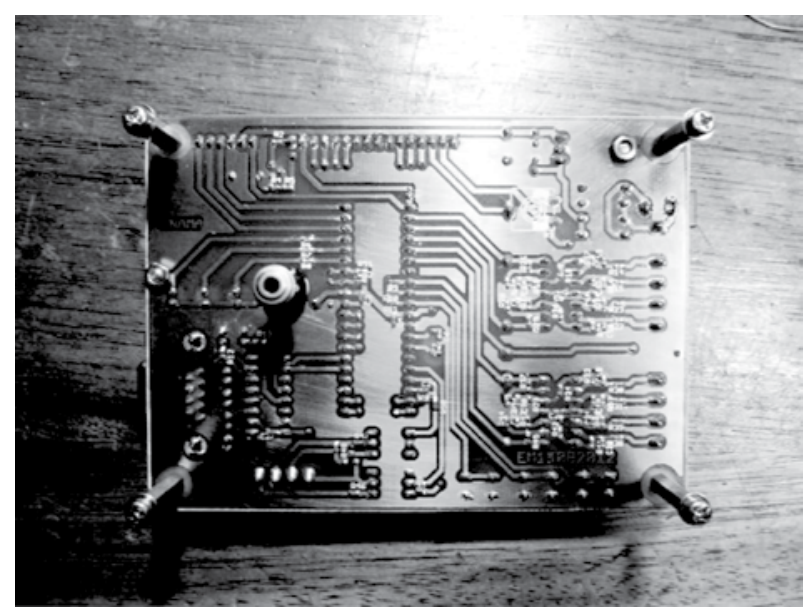

Fuente: Elaboración Propia

Figura 13. Conector Serial de la tarjeta integradora

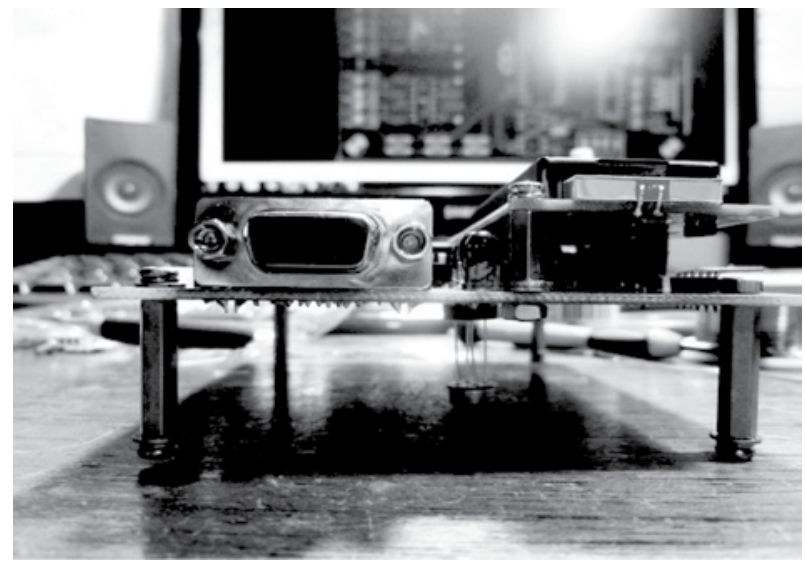

Fuente: Elaboración Propia

\section{CONCLUSIONES}

1. Es posible integrar los módulos de control de procesos del Centro de Manufactura Avanzada, mediante la construcción de una interfaz de comunicaciones basada en microcontroladores PIC.

2. El concentrador eSAT USB 2.0 permite que los módulos de Caudal, Presión y Temperatura del CEMA puedan operar de manera serial y USB dependiendo de la etapa correspondiente

3. A pesar de que nuestro sistema integrador utiliza un microcontrolador PIC, es posible controlar 3 Controladores Lógico programables (PLC) pudiendo realizar etapas más complejas y a menor costo.

\section{REFERENCIAS BIBLIOGRAFÍAS}

[1] Poma, J., Tello, R., Ruiz, E. (2007). Diseño de una estación virtual para el control de las perturbaciones que afectan la temperatura de los procesos industriales. Industrial Data. Vol 7, $\mathrm{N}^{\circ} 1: 33-41$

http://www.ptindustrial.org/index.php/grupos/ home?item=1 (visitado el 20 de Agosto de 2012)

[2] Sociedad Inducontrol S.A.C. (2005) Manual del Módulo de Caudal

[3] Sociedad Inducontrol S.A.C. (2005) Manual del Módulo de Presión

[4] Sociedad Inducontrol S.A.C. (2005) Manual del Módulo de Temperatura 